\title{
ESTUDIO CLÍNICO Y MICROBIOLÓGICO DE LA INFECCIÓN URINARIA ASOCIADA A CATÉTER, EN LOS SERVICIOS DE MEDICINA INTERNA DE UN HOSPITAL UNIVERSITARIO VENEZOLANO
}

\author{
Pedro Quijada-Martínez ${ }^{1, a}$, Ana Flores-Carrero ${ }^{2,3, b}$, Indira Labrador ${ }^{4, c}$, María Araque $^{4, d}$
}

\begin{abstract}
RESUMEN
Objetivos. Determinar las características clínicas y microbiológicas de las infecciones del tracto urinario asociadas a catéter (ITUAC) en pacientes hospitalizados en los servicios de Medicina Interna del Hospital Universitario de Los Andes (HULA), Mérida, Venezuela y establecer la distribución clonal de Enterobacteriaceae multirresistentes productoras de esta infección. Materiales y métodos. Se estudiaron 73 pacientes adultos con cateterismo vesical, durante enero a julio de 2015. El procesamiento microbiológico de las muestras de orina se realizó por métodos convencionales y automatizados. Las $\beta$-lactamasas de espectro extendido (BLEE) y carbapenemasas fueron detectadas fenotípicamente. La tipificación clonal se determinó por la amplificación de secuencias repetitivas por PCR. Resultados. El 53,4\% de los pacientes eran varones, con una edad media de 50,6 años. El promedio de permanencia del catéter fue de 10,9 \pm 6,5 días/paciente. El $54,8 \%$ de los pacientes tuvo urocultivos positivos. Las levaduras fueron el principal agente etiológico (44,7\%), seguido por las enterobacterias (29,8\%). Las enterobacterias, Pseudomonas aeruginosa y Acinetobacter baumannii produjeron BLEE y carbapenemasas asociadas a otros marcadores de resistencia. Dos grupos clonales fueron identificados en cepas de E. coli y K. pneumoniae multirresistentes, los cuales circularon en la unidad de trauma shock de la emergencia de adulto. Conclusiones. Los hallazgos de este estudio permiten evidenciar la necesidad de adoptar estrictos criterios que justifiquen el uso del catéter vesical y la duración del mismo, así como el implementar programas para prevenir y controlar la diseminación de clonas bacterianas multirresistentes en pacientes con ITUAC en los servicios de medicina interna del HULA.
\end{abstract}

Palabras clave: Infecciones urinarias; Catéteres urinarios; Análisis microbiológico; beta-Lactamasas (fuente: DeCS BIREME).

\section{CLINICAL AND MICROBIOLOGICAL STUDY OF CATHETER-ASSOCIATED URINARY TRACT INFECTIONS IN INTERNAL MEDICINE SERVICES OF A VENEZUELAN UNIVERSITY HOSPITAL}

\begin{abstract}
Objectives. To determine the clinical and microbiological characteristics of catheter-associated urinary tract infections (CA-UTI) in patients admitted to the Internal Medicine services of the Hospital Universitario de Los Andes (HULA), Mérida, Venezuela and to establish the clonal distribution of multi-resistant Enterobacteriaceae that produce this infection. Materials and Methods. Seventy-three adult patients with bladder catheterization were studied between January and July 2015. The microbiological processing of the urine samples was performed using conventional and automatized methods. Extendedspectrum beta-lactamase (ESBL) and carbapenemase were detected phenotypically. Clonal classification was determined using repetitive element sequence-based PCR. Results. A total of $53.4 \%$ of the patients were male, and the average age was 50.6 years. The average time the catheter remained in the patient was $10.9 \pm 6.5$ days and $54.8 \%$ of patients had positive urine cultures. Yeasts were the main etiological agent (44.7\%), followed by enterobacteria (29.8\%). Enterobacteria, Pseudomonas aeruginosa, and Acinetobacter baumannii produced ESBL and carbapenemase associated with other resistance markers. Two clonal groups were identified in multi-resistant Escherichia coli and Klebsiella pneumoniae strains that circulated in the shock trauma unit in the adult emergency department. Conclusions. The findings in this study show the need to adopt strict criteria justifying the use of bladder catheterization and its duration, as well as the implementation of programs to prevent and control the spread of multi-resistant bacterial clones in patients with CA-UTI in the HULA Internal Medicine department.
\end{abstract}

Key words: Urinary tract infections; Urinary catheters; Microbiological analysis; beta-Lactamases (source: MeSH NLM).

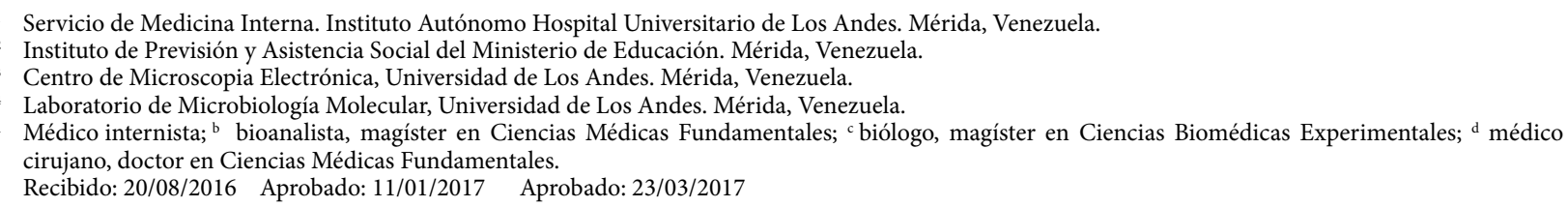

Citar como: Quijada-Martínez P, Flores-Carrero A, Labrador I, Araque M. Estudio clínico y microbiológico de la infección urinaria asociada a catéter en los servicios de medicina interna de un hospital universitario venezolano. Rev Peru Med Exp Salud Publica. 2017;34(1):52-61. doi: 10.17843/rpmesp.2017.341.2766 


\section{INTRODUCCIÓN}

Las infecciones del tracto urinario (ITU) ocupan aproximadamente el $45 \%$ del total de las enfermedades relacionadas con los cuidados de la salud y ocurren en aproximadamente cuatro de cada diez pacientes hospitalizados en todo el mundo ${ }^{(1,2)}$. Un $80 \%$ de estas infecciones están relacionadas con el uso de catéteres vesicales ${ }^{(2)}$. Se estima que entre el 15 y $25 \%$ de los pacientes hospitalizados en los servicios de medicina interna y el $85 \%$ de los ingresados en las unidades de cuidado intensivo son portadores de sondas urinarias durante corto o mediano plazo ${ }^{(3)}$. De esta forma, el riesgo de ITU se incrementa entre 3 y $10 \%$ por cada día de cateterización, alcanzando una probabilidad de infección del $100 \%$ a los 30 días de permanencia del catéter (1-3). En el 2009, el Centers for Desease Control and Prevention (CDC) y el National Healthcare Safety Network (NHSN) señalaron que la presencia de síntomas, niveles de bacteriuria entre $\geq 10^{3} y \leq 10^{5}$ UFC/mL de orina y un uroanálisis positivo, son criterios válidos para el diagnóstico de ITU asociadas a catéter

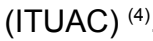

Una amplia variedad de microorganismos son aislados de pacientes con ITUAC. La mayoría de estos proceden principalmente de la flora endógena del paciente, la cual es modificada con frecuencia por la presión antibiótica y la transmisión cruzada por las manos del personal de salud y la exposición a soluciones, equipos y materiales contaminados ${ }^{{ }^{(5)}}$. Los patógenos pueden acceder al tracto urinario por vía extraluminal colonizando la parte externa del dispositivo, o por vía intraluminal donde los microorganismos ingresan a la luz del catéter desde los sitios de conexión y drenaje ${ }^{(3,5)}$. La infección inicial del paciente con cateterismo de corta duración suele ser monomicrobiana y es causada, generalmente, por miembros de la familia Enterobacteriaceae, predominando, en la mayoría de los casos, Escherichia coli ${ }^{(1-3)}$. Cuando el cateterismo es prolongado, las ITU suelen ser polimicrobianas aumentando la frecuencia de Klebsiella spp., Proteus spp., Pseudomonas aeruginosa, especies de Enterococcus y Candida spp. ${ }^{(3,5)}$. Cabe destacar que los pacientes cateterizados son un reservorio importante de microorganismos multirresistentes, entre los que se incluyen bacterias Gram negativas productoras de $\beta$-lactamasas de espectro extendido (BLEE) y carbapenemasas, lo cual dificulta el tratamiento empírico cuando la ITU es grave ${ }^{(1,3,5)}$.

A pesar de que el tratamiento de las ITUAC no es fácil, debido a que la evolución de esta infección puede derivar en cuadros crónicos o en infecciones recurrentes, el diagnóstico temprano a través de criterios clínicos y paraclínicos, así como la identificación del agente etiológico y la aplicación de una terapia antibiótica guiada con base a las pruebas de susceptibilidad, son fundamentales para evitar las complicaciones y mejorar el pronóstico del paciente ${ }^{(3,5,6)}$. Desafortunadamente, en Venezuela no existen registros sobre el perfil microbiológico ni se conocen los patrones de resistencia antimicrobiana de los agentes productores ITUAC en hospitales públicos. Por tal motivo, el objetivo de este estudio fue determinar las características clínicas y microbiológicas de las ITUAC en pacientes hospitalizados en los servicios de medicina interna del Hospital Universitario de Los Andes (HULA), Mérida, Venezuela y, además, establecer la distribución clonal de Enterobacteriaceae multirresistentes productoras de esta infección.

\section{MATERIALES Y MÉTODOS}

Este estudio fue de tipo observacional, de corte transversal y descriptivo y se realizó durante enero a junio de 2015.

\section{PACIENTES}

Se seleccionaron 73 pacientes adultos de ambos sexos, a quienes se les practicó cateterismo vesical durante la hospitalización en las áreas del servicio de Medicina Interna del HULA.

\section{CRITERIOS DE INCLUSIÓN Y EXCLUSIÓN}

Los pacientes cumplieron con los criterios de inclusión siguientes: motivo de consulta y hospitalización diferente a ITU con uroanálisis sin alteraciones, cateterización mayor a $48 \mathrm{~h}$ y presencia de signos y síntomas de ITU, así como uroanálisis patológico posterior a la colocación del catéter vesical. Se excluyeron de este estudio los pacientes con cirugía urológica, embarazadas y personas que manifestaran su rechazo a la participación de la investigación.

\section{VARIABLES DE ESTUDIO}

En este trabajo, la ITUAC fue definida de acuerdo a lo descrito por el CDC/NHSN ${ }^{(4)}$. Las variables estudiadas estuvieron constituidas por los datos clínicos y epidemiológicos de cada paciente, los cuales fueron registrados en una ficha diseñada para los efectos de este estudio.

\section{RECOLECCIÓN DE LA MUESTRA CLÍNICA}

Previa asepsia y antisepsia de los genitales externos, a todos los pacientes incluidos en este estudio se les cambió la sonda vesical antes de la recolección de la muestra de orina. La muestra fue colectada $(10 \mathrm{~mL})$ por 
aspiración con una jeringa estéril a través de la sonda, transferida a un colector estéril y procesada de forma inmediata en el laboratorio.

\section{PROCESAMIENTO MICROBIOLÓGICO DE LAS MUESTRAS DE ORINA}

Inicialmente, se realizó el estudio macro y microscópico (tinción de Gram) y mediante la técnica del asa calibrada se procedió a inocular placas con los medios de cultivo (BBL ${ }^{\mathrm{TM}}$, Becton Dickinson, Cockeysville, MD, USA): sangre, MacConkey y manitol-salado. El criterio que se utilizó para considerar ITU en pacientes cateterizados fue; $\geq 10^{3} \mathrm{UFC} / \mathrm{mL}$. Todos los microorganismos aislados fueron identificados mediante el sistema VITEK $^{\circledR} 2$ Technology (BioMérieux, Marcy L'Etoile, France) y las pruebas de susceptibilidad antimicrobiana se realizaron determinando la concentración inhibitoria mínima utilizando las tarjetas VITEK 2 AST-N299, AST-N298 y AST-YS07 para bacterias Gram negativas, Gram positivas y levaduras, respectivamente.

\section{DETERMINACIÓN FENOTÍPICA DE $\beta$-LACTAMASAS DE ESPECTRO EXTENDIDO (BLEE) Y CARBAPENEMASAS}

La detección de BLEE se realizó utilizando la prueba del sinergismo del doble disco (SDD) de acuerdo al Clinical and Laboratory Standards Institute (CLSI) ${ }^{(7)}$. Se utilizaron E. coli ATCC 25922 (BLEE negativo) y E. coli LMM-26 (BLEE positiva) como controles.

La detección fenotípica de carbapenemasas se realizó, inicialmente, mediante el método modificado de Hodge, descrito en el CLSI (7) y, posteriormente, en los casos que resultaron positivos se les determinó la presencia de metalo $\beta$-lactamasas utilizando discos de imipenem $(10 \mu \mathrm{g})$, meropenem $(10 \mu \mathrm{g})$ yácido etilendiaminotetracéticomercaptoacético de sodio (EDTA-SMA) (Sigma-Aldrich, St Louis, MO, USA), de acuerdo con Guevara et al. (8). Se utilizó $P$. aeruginosa $77297\left(b / a_{\mathrm{VIM}}\right)$ como control. Para la detección de la enzima Klebsiella pneumoniae-carbapenemasa (KPC) se utilizaron discos de ácido borónico (BBL) y ertapenem $(10 \mu \mathrm{g})$. En esta prueba se utilizó como control positivo $K$. oxytoca LMM-SA26 (bla KPC-2 $)$.

\section{TIPIFICACIÓN DE CEPAS Enterobacteriaceae POR $R E P-P C R$}

La relación clonal entre las cepas fue determinada por la amplificación de secuencias repetitivas por PCR (RepPCR) a partir del ADN total, utilizando los iniciadores: REP-1 F: 5` IIIGCGCCGICATCAGGC - 3`; REP-2 R: 5`- ACGTCTTATCAGGCCTAC - 3`y las condiciones descritas por Lozano et al. (9). Los productos de amplificación se separaron en un gel de agarosa al 1,5\%, teñido con bromuro de etidio y fotografiados con el UVP
Biodoc-It System. Los resultados obtenidos del RepPCR fueron analizados utilizando el software Treecon 1.3b. Los patrones con coeficientes de similitud superior al $90 \%$ fueron considerados relacionados clonalmente.

\section{ASPECTOS ÉTICOS}

Este estudio fue aprobado por el comité de bioética de la Facultad de Medicina y del Consejo de Desarrollo Científico, Humanístico, Tecnológico y de las Artes de la Universidad de Los Andes, bajo el código No. FA572-14-07-EE. En todos los casos, y previo a la toma de muestras, se obtuvo el consentimiento informado de los pacientes.

\section{RESULTADOS}

En la tabla 1 se resumen las características clínicas y epidemiológicas de los pacientes estudiados. Los 73 pacientes se distribuyeron en $34(46,6 \%)$ femeninos y $39(53,4 \%)$ masculinos, con un promedio de edad de 50,6 años y un rango entre 16 y 96 años. Los grupos etarios de 21 a 40 y 61 a 80 años concentraron el mayor número de pacientes en igual proporción (23/73; 31,5\%, cada uno), observándose un predominio del sexo masculino (21,9 y $16,4 \%$, respectivamente). El $86,3 \%$ $(63 / 73)$ de los pacientes se encontraban hospitalizados en el servicio de emergencia, distribuidos de la siguiente manera: $61,6 \%$ en la unidad de trauma shock, seguidos por los admitidos en observación mixta con un $23,3 \%$, en tanto que el 1,4\% se ubicó en el área de estabilización. El resto de los pacientes estudiados (10/73; 13,7\%) estaban recluidos en la hospitalización general de adultos (salas T4, T5 y T6). Ocho diagnósticos de admisión fueron registrados, siendo los más frecuentes en los pacientes masculinos el trauma craneoencefálico y politraumatizados, junto con accidentes cerebro vascular $(\mathrm{ACV})$ isquémico o hemorrágico, mientras que las enfermedades de tipo infeccioso fueron las predominantes en el sexo femenino. Los días de permanencia del catéter oscilaron entre 2 a 30 días, con un promedio de 10,9 \pm 6,5 días, siendo de mayor duración en los pacientes masculinos (12,5 \pm 7,5 días).

El $52,1 \%$ de los pacientes estaba bajo tratamiento con antimicrobianos, de manera que para el momento de la toma de la muestra el $42,5 \%$ recibía, por lo menos, un antibiótico. Las cefalosporinas de tercera generación, las flouroquinolonas y los carbapenemos fueron los antibióticos prescritos con mayor frecuencia. La monoterapia estuvo representada por ampicilina/ sulbactam seguida de ceftriaxona. Las combinaciones más frecuentes fueron: ceftriaxona + levofloxacino, meropenem + metronidazol e imipenem + metronidazol y la triple terapia se observó con el uso combinado de imipenem + clindamicina + fluconazol (Tabla 1). 
Tabla 1. Características clínico-epidemiológicas de los pacientes con ITUAC

\begin{tabular}{|c|c|c|c|c|c|c|}
\hline \multirow[t]{2}{*}{ Característica } & \multicolumn{2}{|c|}{$\begin{array}{c}\text { Femenino } \\
34(46,6 \%) \\
\end{array}$} & \multicolumn{2}{|c|}{$\begin{array}{l}\text { Masculino } \\
39(53,4 \%) \\
\end{array}$} & \multicolumn{2}{|c|}{$\begin{array}{c}\text { Total } \\
73(100 \%) \\
\end{array}$} \\
\hline & $\mathbf{n}$ & $\%$ & $\mathbf{n}$ & $\%$ & $\mathbf{n}$ & $\%$ \\
\hline \multicolumn{7}{|l|}{ Grupo etario (años) } \\
\hline$<20$ & 4 & 5,5 & 3 & 4,1 & 7 & 9,6 \\
\hline $21-40$ & 7 & 9,6 & 16 & 21,9 & 23 & 31,5 \\
\hline $41-60$ & 7 & 9,6 & 7 & 9,6 & 14 & 19,2 \\
\hline $61-80$ & 11 & 15,1 & 12 & 16,4 & 23 & 31,5 \\
\hline$\geq 81$ & 5 & 6,8 & 1 & 1,4 & 6 & 8,2 \\
\hline \multicolumn{7}{|l|}{ Área de hospitalización } \\
\hline \multicolumn{7}{|l|}{ Emergencia de adultos } \\
\hline Trauma Shock & 21 & 28,8 & 24 & 32,9 & 45 & 61,6 \\
\hline Estabilización & 0 & 0 & 1 & 1,4 & 1 & 1,4 \\
\hline Observación Mixta & 7 & 9,6 & 10 & 13,6 & 17 & 23,3 \\
\hline \multicolumn{7}{|l|}{ Hospitalización general adultos } \\
\hline Sala T4 & 0 & 0 & 2 & 2,7 & 2 & 2,7 \\
\hline Sala T5 & 5 & 6,8 & 2 & 2,7 & 7 & 9,6 \\
\hline Sala T6 & 1 & 1,4 & 0 & 0 & 1 & 1,4 \\
\hline \multicolumn{7}{|l|}{ Diagnóstico de ingreso } \\
\hline Patología traumática severa * & 6 & 8,2 & 17 & 23,3 & 23 & 31,5 \\
\hline ACV isquémico/hemorrágico & 4 & 5,5 & 8 & 11 & 12 & 16,5 \\
\hline Sepsis/shock séptico & 9 & 12,4 & 2 & 2,7 & 11 & 15,1 \\
\hline Abdomen agudo médico/quirúrgico & 6 & 8,2 & 4 & 5,5 & 10 & 13,7 \\
\hline Patología coronaria aguda & 3 & 4,1 & 1 & 1,4 & 4 & 5,5 \\
\hline Diabetes mellitus & 3 & 4,1 & 1 & 1,4 & 4 & 5,5 \\
\hline Urgencias neurológicas no vasculares & 2 & 2,7 & 2 & 2,7 & 4 & 5,4 \\
\hline Otros & 1 & 1,4 & 4 & 5,4 & 5 & 6,8 \\
\hline \multicolumn{7}{|l|}{ Duración del catéter (días) } \\
\hline 5 & 11 & 15,1 & 4 & 5,4 & 15 & 20,5 \\
\hline $6-12$ & 12 & 16,4 & 24 & 32,9 & 36 & 49,3 \\
\hline $13-19$ & 6 & 8,2 & 8 & 11 & 14 & 19,2 \\
\hline $20-26$ & 4 & 5,5 & 2 & 2,7 & 6 & 8,2 \\
\hline$\geq 27$ & 1 & 1,4 & 1 & 1,4 & 2 & 2,8 \\
\hline \multicolumn{7}{|l|}{ Terapia antibiótica inicial } \\
\hline Ninguno & 17 & 23,3 & 18 & 24,7 & 35 & 47,9 \\
\hline Monoterapia & 13 & 17,8 & 18 & 24,7 & 31 & 42,5 \\
\hline Dos antibióticos & 3 & 4,1 & 3 & 4,1 & 6 & 8,2 \\
\hline Más de dos antibióticos & 1 & 1.4 & 0 & 0 & 1 & 1,4 \\
\hline \multicolumn{7}{|l|}{ Antibiótico recibido previamente } \\
\hline Monobactámicos & 1 & 1,1 & 2 & 2,2 & 3 & 3,3 \\
\hline Cefalosporinas de primera generación & 1 & 1,1 & 0 & 0 & 1 & 1,1 \\
\hline Cefalosporinas de tercera generación & 11 & 12 & 8 & 8,7 & 19 & 20,7 \\
\hline Cefalosporinas de cuarta generación & 0 & 0 & 1 & 1,10 & 1 & 1,1 \\
\hline$\beta$-lactámico/inhibidor $\beta$-lactamasa & 4 & 4,4 & 7 & 7,6 & 11 & 12,0 \\
\hline Carbapenemos & 8 & 8,7 & 6 & 6,5 & 14 & 15,2 \\
\hline Fluoroquinolonas & 10 & 10,9 & 6 & 6,5 & 16 & 17,4 \\
\hline Aminoglucósidos & 0 & 0 & 3 & 3,3 & 3 & 3,3 \\
\hline Colistina & 0 & 0 & 1 & 1,1 & 1 & 1,1 \\
\hline Clindamicina & 1 & 1,1 & 2 & 2,2 & 3 & 3,3 \\
\hline Linezolid & 0 & 0 & 1 & 1,1 & 1 & 1,1 \\
\hline Vancomicina & 1 & 1,1 & 5 & 5,4 & 6 & 6,5 \\
\hline Metronidazol & 8 & 8,7 & 3 & 3,3 & 11 & 12 \\
\hline \multicolumn{7}{|l|}{ Cultivo microbiano } \\
\hline Negativo & 15 & 20,5 & 18 & 24,7 & 33 & 45,2 \\
\hline Positivo & 19 & 26,0 & 21 & 28,8 & 40 & 54,8 \\
\hline
\end{tabular}

* Incluye trauma craneoencefálico y politraumatizados; ACV: accidente cerebro vascular. ITUAC: infección del tracto urinario asociadas a catéter. 
El $54,8 \%(40 / 73)$ de los pacientes tuvo urocultivos positivos. En la tabla 2 se describen los microorganismos aislados y sus distintas asociaciones. Del total de 47 microorganismos identificados, las levaduras fueron el principal agente etiológico aislado (21/47; 44,7\%), seguido por las enterobacterias con 29,8\% (14/47); en tercer lugar, las bacterias Gram positivas con $14,9 \%(7 / 47)$ y en menor frecuencia los bacilos Gram negativos no fermentadores con 10,6\% (5/47). El cultivo monomicrobiano fue el resultado más frecuente (34/40; $85 \%)$, donde C. tropicalis (8/34), C. albicans (6/34), E. coli $(5 / 34)$, K. pneumoniae (4/34) y E. faecalis $(3 / 34)$ ocuparon en frecuencia los primeros cinco lugares. Los cultivos mixtos representaron el $12,5 \%$ (5/40) y en todos, las especies de Candida estuvieron asociadas a diferentes bacterias Gram negativas o Gram positivas. El único cultivo polimicrobiano estuvo conformado por tres bacterias Gram negativas (2,5\%; $1 / 40)$.

Tabla 2. Microorganismos aislados en pacientes con ITUAC y patrones de asociación microbiana

\begin{tabular}{|c|c|c|}
\hline Característica & $\mathbf{n}$ & $\%$ \\
\hline \multicolumn{3}{|l|}{ Microorganismo aislado $(n=47)$} \\
\hline Levaduras & 21 & 44,7 \\
\hline Enterobacterias & 14 & 29,8 \\
\hline Cocos Gram-positivos & 7 & 14,9 \\
\hline $\begin{array}{l}\text { Bacilos Gram-negativos no } \\
\text { fermentadores }\end{array}$ & 5 & 10,6 \\
\hline \multicolumn{3}{|l|}{ Cultivos positivos $(n=40)$} \\
\hline Monomicrobianos & 34 & 85,0 \\
\hline Candida tropicalis & 8 & 23,5 \\
\hline Candida albicans & 6 & 17,7 \\
\hline Escherichia coli & 5 & 14,7 \\
\hline Klebsiella pneumoniae & 4 & 11,8 \\
\hline Enterococcus faecalis & 3 & 8,8 \\
\hline Enterococcus faecium & 1 & 2,9 \\
\hline Complejo Enterobacter clocae & 1 & 2,9 \\
\hline Complejo Acinetobacter baumannii & 1 & 2,9 \\
\hline Stenotrophomonas maltophilia & 1 & 2,9 \\
\hline Staphylococcus epidermidis & 1 & 2,9 \\
\hline Staphylococcus aureus & 1 & 2,9 \\
\hline Candida famata & 1 & 2,9 \\
\hline Crytococcus laurentii & 1 & 2,9 \\
\hline Asociación de dos microorganismo & 5 & 12,5 \\
\hline $\begin{array}{l}\text { Candida tropicalis + Pseudomonas } \\
\text { aeruginosa }\end{array}$ & 1 & 20,0 \\
\hline $\begin{array}{l}\text { Candida parapsilosis + Enterococcus } \\
\text { faecium }\end{array}$ & 1 & 20,0 \\
\hline Candida albicans + Escherichia coli & 1 & 20,0 \\
\hline Candida glabrata + Escherichia coli & 1 & 20,0 \\
\hline $\begin{array}{l}\text { Candida glabrata + Pseudomonas } \\
\text { aeruginosa }\end{array}$ & 1 & 20,0 \\
\hline Asociación de tres microorganismo & 1 & 2,5 \\
\hline $\begin{array}{l}\text { Pseudomonas aeruginosa + Escherichia } \\
\text { coli + Klebsiella pneumoniae }\end{array}$ & 1 & 100 \\
\hline
\end{tabular}

ITUAC: infección del tracto urinario asociadas a catéter.
Todas las levaduras fueron sensibles a los seis antifúngicos probados, solo una cepa de $C$. tropicalis mostró resistencia a flucitosina. Diversos perfiles de multirresistencia fueron observados en las bacterias aisladas (Tabla 3). E. coli fue la enterobacteria con mayor número de marcadores de resistencia, en el que se incluyeron cefalosporinas de amplio espectro y carbapenemos. Un patrón similar fue observado en $P$. aeruginosa y $A$. baumannii. Todas las cepas Gram negativas presentaron resistencia asociada a las quinolonas y, por lo menos, a un representante de los aminoglucósidos. En las bacterias Gram positivas, la resistencia a los $\beta$-lactámicos, linezolid, gentamicina y

Tabla 3. Patrones de resistencia de las bacterias aisladas de pacientes con ITUAC

\begin{tabular}{|c|c|}
\hline Bacteria & \multirow{2}{*}{$\mathbf{n}$} \\
\hline Patrón de resistencia & \\
\hline \multicolumn{2}{|l|}{ Escherichia coli $(\mathrm{n}=8)$} \\
\hline $\begin{array}{l}\text { Amp, Ptz, Sam, Ctx, Ctz, Azt, Ert, Imp, Mer, Amk, } \\
\text { Gtm, Acn, Cip. }\end{array}$ & 5 \\
\hline Amp, Ptz, Sam, Ctx, Ctz, Azt, Gtm, Acn, Cip & 1 \\
\hline Amk, Gtm, Acn, Cip & 2 \\
\hline \multicolumn{2}{|l|}{ Klebsiella pneumoniae $(n=5)$} \\
\hline $\begin{array}{l}\text { Ptz, Sam, Ctx, Ctz, Azt, Ert, Imp, Mer, Gtm, Acn, } \\
\text { Cip. }\end{array}$ & 3 \\
\hline Ptz, Sam, Ctx, Ctz, Azt, Amk, Gtm, Acn, Cip. & 2 \\
\hline \multicolumn{2}{|l|}{ Complejo Enterobacter cloacae $(\mathrm{n}=1)$} \\
\hline Ptz, Sam, Ctx, Ctz, Azt, Amk, Acn, Cip. & 1 \\
\hline \multicolumn{2}{|l|}{ Pseudomonas aeruginosa $(n=3)$} \\
\hline Ctx, Cfz, Ert, Imp, Mer, Amk, Gtm, Cip, Lvx & 2 \\
\hline Pip, Amk, Gtm, Cip, Lvx & 1 \\
\hline \multicolumn{2}{|l|}{ Complejo Acinetobacter baumannii $(n=1)$} \\
\hline Ctx, Cfz, Ert, Imp, Mer, Amk, Gtm, Cip, Lvx & 1 \\
\hline \multicolumn{2}{|l|}{ Stenotrophomonas maltophilia $(n=1)$} \\
\hline Cip, Lvx, Stx & 1 \\
\hline \multicolumn{2}{|l|}{ Enterococcus faecalis $(n=3)$} \\
\hline Amp, Eri, Cln, Lnz, Cip, Lvx & 3 \\
\hline \multicolumn{2}{|l|}{ Enterococcus faecium $(n=2)$} \\
\hline Amp, Eri, Cln, Gtm, Qnp/DIf, Lnz, Cip, Lvx & 1 \\
\hline \multicolumn{2}{|l|}{ Amp, Eri, Cln, Gtm, Lnz, Cip, Lvx } \\
\hline \multicolumn{2}{|l|}{ Staphylococcus epidermidis $(\mathrm{n}=1)$} \\
\hline Oxa, Eri, Cln, Gtm, Qnp/DIf, Lnz, Cip, Lvx & 1 \\
\hline \multicolumn{2}{|l|}{ Staphylococcus aureus $(n=1)$} \\
\hline Oxa, Gtm, Lnz, Cip, Lvx & 1 \\
\hline
\end{tabular}

Amp: Ampicilina, Oxa: oxacilina; Eri: eritromicina; CIn: clindamicina; Sam: ampicilina/sulbactam; Ptz: piperacilina/tazobactam; Ctz: ceftazidima; Ctx: cefotaxima; Azt: aztreonam: Imp: Imipenem; Ert: ertapenem; Mem: meropenem; Amk: amikacina; Gem: gentamicina; Acn: ácido nalidixico; Cip: ciprofloxacin; Lvx: levofloxacina; Mxf: maxifloxacina; Qda: quinupristin dalfopristina; Lnz: linezolid; Sxt: trimetroprim/sulfametoxazole. ITUAC: infección del tracto urinario asociadas a catéter. 
fluoroquinolonas fue una característica común y en la mayoría de los casos, este fenotipo se asoció con una pobre susceptibilidad a macrólidos y lincosaminas. Una cepa de E. faecium y otra de Staphylococcus epidermidis presentaron resistencia al quinuspritina/dalfopristina. Colistina y tigeciclina fueron los antibióticos con mayor actividad sobre las bacterias Gram negativas. En los Gram positivos, además de tigeciclina, también fueron sensibles a glucopéptidos y tetraciclinas.

En la tabla 4, se resumen las características epidemiológica, fenotípicas y la distribución clonal de las cepas Gram negativas productoras de $\beta$-lactamasas. Todas las enterobacterias fueron productoras de BLEE y ocho de estas coprodujeron carbapenemasas. Tres E. coli y una K. pneumoniae fueron positivas para KPC y en las otras cuatro restantes se les detectó metalo$\beta$-latamasas. Todas las cepas de $P$. aeruginosa y $A$. baumannii fueron positivas para metaloenzimas. Las cepas productoras de $\beta$-lactamasas predominaron en pacientes femeninas mayores de 40 años, con permanencia del catéter no menor a 10 días.

Dos grupos clonales ( $\mathrm{A}$ y $\mathrm{B}$ ) fueron observados en las cepas de E. coli. El clonal A concentró la mayoría de las cepas (5/6). Esta se subdividió en A1 con 3 cepas con una relación genética de $90 \%$ y el A2 integrado por 2 cepas de $E$. coli con aproximadamente $95 \%$ de similitud. La cepa LMM-1194.3 constituyó la clona B con menos del $20 \%$ de relación con los grupos A1 y A2 (Fig 8 AB y tabla 4). K. pneumoniae se distribuyó en dos grupos bien definidos (I y II), conformados por cepas con $100 \%$ de similitud (Figura 1. C y D; Tabla 4). Los grupos clonales A1 y B de E. coli y el IA de K. pneumoniae se observaron con mayor frecuencia en pacientes hospitalizados en trauma shock. El A2 se detectó solo en pacientes recluidos en la sala $T 5$ de hospitalización general y la clona IIA en observación mixta.

Tabla 4. Características epidemiológicas y distribución clonal de las cepas Gram negativas productoras de $\beta$-lactamasas en pacientes con ITUAC

\begin{tabular}{|c|c|c|c|c|c|c|}
\hline $\begin{array}{l}\text { Código } \\
\text { de cepa }\end{array}$ & $\begin{array}{c}\text { Edad del } \\
\text { paciente } \\
\text { (años) }\end{array}$ & Sexo & $\begin{array}{l}\text { Permanencia } \\
\text { del catéter } \\
\text { (días) }\end{array}$ & $\begin{array}{c}\text { Área de } \\
\text { hospitalización }\end{array}$ & $\begin{array}{l}\text { Presencia de } \\
\beta \text {-lactamasa }\end{array}$ & $\begin{array}{c}\text { Patrón } \\
\text { clonal } \\
\text { Rep-PCR }\end{array}$ \\
\hline \multicolumn{7}{|l|}{ Escherichia coli } \\
\hline LMM-1147 & 73 & $\mathrm{~F}$ & 18 & Obsv. mixta & BLEE + MßL & A1 \\
\hline LMM-1195 & 43 & $\mathrm{~F}$ & 19 & Trauma shock & BLEE + MßL & A1 \\
\hline LMM-1199 & 68 & $\mathrm{~F}$ & 10 & Trauma shock & BLEE & A1 \\
\hline LMM-15131.1 & 53 & $\mathrm{~F}$ & 13 & Sala T5 & $\mathrm{BLEE}+\mathrm{KPC}$ & A2 \\
\hline LMM-15131.2 & 42 & $\mathrm{~F}$ & 14 & Sala T5 & $\mathrm{BLEE}+\mathrm{KPC}$ & A2 \\
\hline LMM-1194.3 & 34 & M & 8 & Trauma shock & $\mathrm{BLEE}+\mathrm{KPC}$ & B \\
\hline \multicolumn{7}{|c|}{ Klebsiella pneumoniae } \\
\hline LMM-141060 & 71 & $\mathrm{~F}$ & 15 & Trauma shock & BLEE + MßL & IA \\
\hline LMM-1194.2 & 34 & M & 8 & Trauma shock & BLEE & $\mathrm{IA}$ \\
\hline LMM-14195 & 19 & M & 10 & Trauma shock & $B L E E+M \beta L$ & IA \\
\hline LMM-524.1 & 71 & $\mathrm{~F}$ & 12 & Obsv. mixta & BLEE & IIA \\
\hline LMM-14524.2 & 71 & $\mathrm{~F}$ & 12 & Obsv. mixta & $\mathrm{BLEE}+\mathrm{KPC}$ & IIA \\
\hline \multicolumn{7}{|c|}{ Enterobacter cloacae } \\
\hline LMM-1064 & 61 & M & 9 & Trauma shock & BLEE & NA \\
\hline \multicolumn{7}{|c|}{ Pseudomona aeruginosa } \\
\hline LMM-1194.3 & 34 & $M$ & 9 & Trauma shock & $M \beta L$ & NA \\
\hline LMM-1195.2 & 43 & $\mathrm{~F}$ & 19 & Trauma shock & $M \beta L$ & NA \\
\hline \multicolumn{7}{|c|}{ Acinetobacter baumannii } \\
\hline LMM-496 & 96 & $\mathrm{~F}$ & 10 & Trauma shock & $\mathrm{M} \beta \mathrm{L}$ & NA \\
\hline
\end{tabular}

* Patrón Rep-PCR: agrupaciones clonales generadas por el análisis del software Treecon 1.3b. Obsv. mixta: observación mixta; BLEE: $\beta$-lactamasa de espectro extendido; KPC: Klebsiella pneumoniae-carbapenemasa; M $\beta$ L : metalo- $\beta$-lactamasa; NA: no aplicable. ITUAC: infección del tracto urinario asociadas a catéter. 


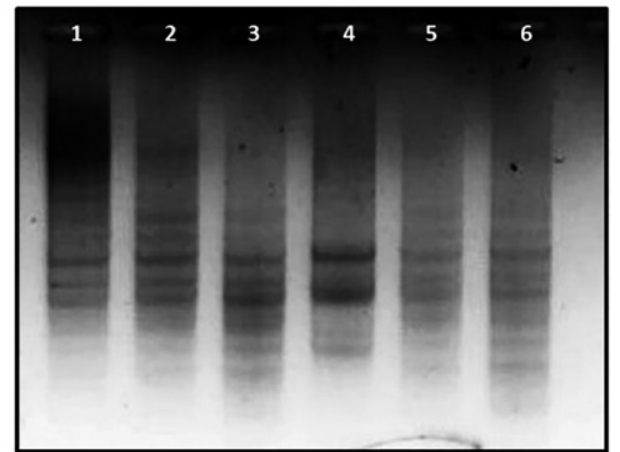

A

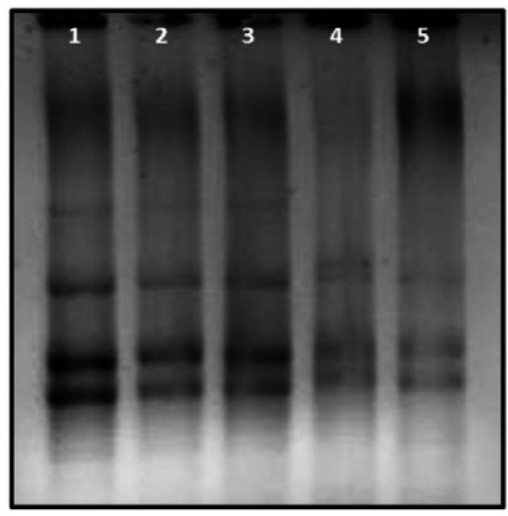

C

D

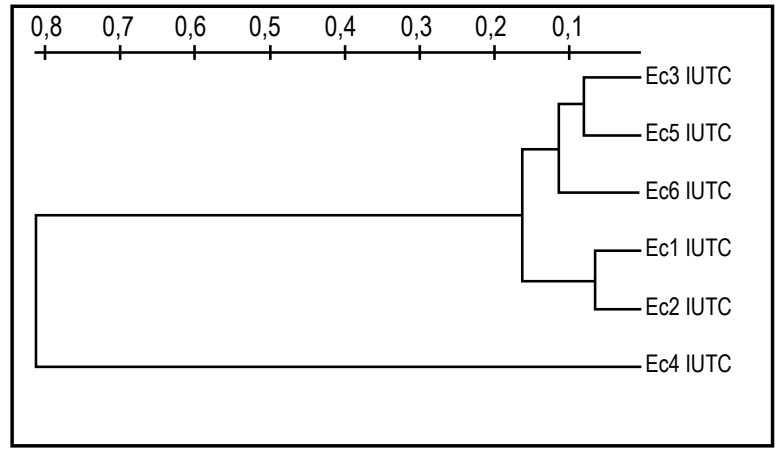

B

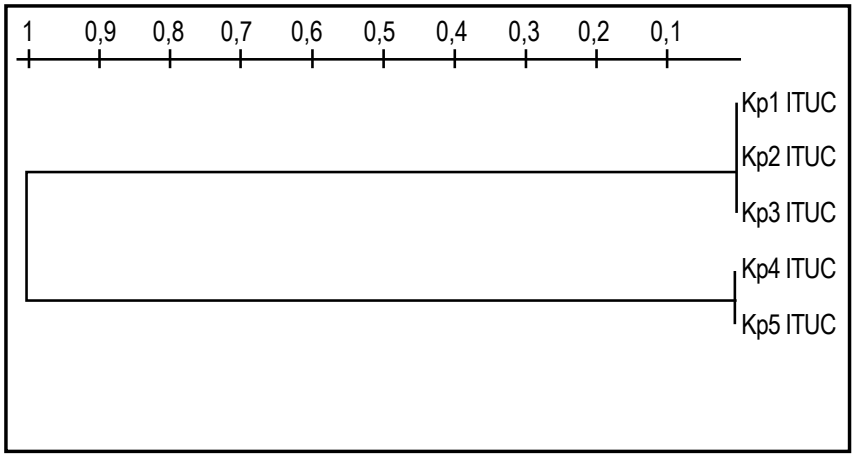

Figura 1. Electroforesis del Rep-PCR (A y C). B: dendograma que muestra la relación clonal de las cepas de $E$. coli productoras de $\beta$-lactamasas. Ec3: LMM-1147; Ec5: LMM-1195; Ec6: LMM-1199; Ec1: LMM-15131.1; Ec2: LMM-15131.2; Ec4: LMM-1194.3. D: dendograma que muestra la relación clonal de las cepas de $K$. pneumoniae productoras de ß-lactamasas Kp1: LMM-141060; Kp2: LMM-1194.2; Kp3: LMM-14195; Kp4: LMM-524.1; Kp5: LMM-14524.2. Cada grado en la escala representa un $10 \%$ de diferencia en el índice de similitud.

\section{DISCUSIÓN}

En este estudio se analizaron 73 pacientes con cateterización urinaria, de estos, más de la mitad $(54,8 \%)$ cursaron con una ITU durante su estancia hospitalaria en los servicios de Medicina Interna del HULA. Esta frecuencia fue mayor a la reportada por De Lira et al. ${ }^{(10)}$ en México, quienes encontraron una incidencia del 28,3 y $34,5 \%$ en pacientes con ITU asociados a sonda de Foley en hospitales del nivel II y III, respectivamente. De igual forma, Duszynska et al. ${ }^{(11)}$ en Polonia, y Dougnon et al. (12) en Benin (West Africa), registran cifras mucho menores de ITUAC (36 y $23,3 \%$, respectivamente) a las descritas en este estudio.

Kanj et al. ${ }^{(13)}$ señalaron que la incidencia de las ITUAC es desestimada en países con recursos limitados, y el uso excesivo de catéteres vesicales en ambientes intrahospitalarios es una práctica común. Al respecto, varios estudios demuestran que entre un 21 y $55,7 \%$ de las indicaciones de colocación de catéteres vesicales en pacientes hospitalizados no están claras $(3,5,6,14)$. Actualmente, el HULA carece de normativas que orienten el uso oportuno y adecuado de los catéteres vesicales, así como protocolos para el cuidado de pacientes cateterizados. Por consiguiente, no sorprende el significativo aumento en la frecuencia de ITUAC en este hospital en comparación con otros centros de salud (10-13).

Las ITUAC fueron más frecuentes en los adultos jóvenes de 21 a 40 y en el grupo etario mayor de 60 años, principalmente en el sexo masculino. Aunque la edad y el sexo se han asociado clásicamente con el desarrollo de ITU no complicadas (1-5), varios autores señalan que en la patogenia de ITUAC son fundamentales dos elementos: la capacidad de adhesión del uropatógeno y la posibilidad de este para formar biopelícula ${ }^{(3,5,15)}$. Algunos estudios han demostrado la presencia de biopelículas a las $24 \mathrm{~h}$ después de la inserción del catéter ${ }^{(5)}$. De manera que el riesgo de desarrollar ITUAC 
se incrementa paulatinamente a medida que se prolongan los días de cateterización. De hecho, en este estudio se demostró que el porcentaje de cultivos positivos aumentó considerablemente de un $2,7 \%$ en pacientes sondeados con menos de 5 días a un 100\% después de 25 días. Al-Hazmi ${ }^{(16)}$ y Liedl (17) reportaron que el $77 \%$ de los pacientes con urocultivos positivos estuvieron expuestos a canalización uretral por más de 8 días. Por otra parte, algunas comorbilidades se han considerado factores clásicamente favorecedores del desarrollo ITUAC (1-3), tales como: diabetes mellitus, enfermedad renal crónica, neoplasias, patologías prostáticas y la neutropenia. No obstante, en este estudio se identificaron otras patologías como el traumatismo severo, ACV y sepsis/shock séptico, las cuales probablemente condicionaron el desarrollo de ITUAC.

El espectro microbiológico que causa ITUAC comúnmente está dominado por miembros de la familia Enterobacteriaceae ${ }^{(3,16,18)}$. En este estudio los cultivos monomicrobianos fueron los más frecuentes, pero al igual que Esquivel et al. (19), Chincha et al. ${ }^{(20)} \mathrm{y}$ Deorukhkar y Saini ${ }^{21)}$ se encontró un predominio de especies de Candida (44,7\%), destacando C. tropicalis como la causa más frecuente de candiduria, dejando en el segundo lugar a las enterobacterias con un $29,8 \%$. Estos resultados marcan una diferencia con los datos aportados por Al-Hazmi (16) y Vyawahare et al. (18) quienes ubican a las levaduras en el tercer lugar de sus aislamientos. Por otra parte, Pigrau (3) refiere que la frecuencia de candiduria por C. albicans, y otras especies, oscila entre el 3 y $20 \%$ y es especialmente frecuente en pacientes ancianos, diabéticos y los sometidos a ciclos de terapias con antibióticos de amplio espectro. Es probable que los resultados obtenidos en este trabajo hayan estado influenciados por el hecho de que $52,1 \%$ de los pacientes estaban bajo tratamiento con antibióticos y, de estos, el 32,9 \% tenían una terapia combinada con una cefalosporina de tercera generación o un carbapenemo. Aunque todas las cepas de Candida aisladas en este estudio presentaron una amplia susceptibilidad a los agentes antifúngicos, en la última década el género Candida han mostrado perfiles de resistencia diferentes de acuerdo a la especie, especialmente al fluconazol ${ }^{(22,23)}$. Esto justifica la necesidad de identificar en forma precisa las levaduras aisladas y evaluar la susceptibidad in vitro previo a una decisión terapéutica.

A pesar de que las bacterias no fueron el principal agente etiológico causantes de las ITUAC en este trabajo, estas mostraron un perfil de resistencia que requirió atención. $E$. coli, $K$. pneumoniae y $E$. cloacae, además de tener resistencia a quinolonas y aminoglucósidos, presentaron un perfil fenotípico compatible con la producción de BLEE y, en algunas cepas, sorprendió la resistencia a carbapenemos. Se ha descrito que el método de Hodge modificado puede tener una baja sensibilidad para la detección de algunas carbapenemasas ${ }^{24)}$. Sin embargo, en este estudio la presencia de estas enzimas fue confirmada mediante la aplicación de los métodos de sinergismo con inhibidores específicos (EDTA-SMA y ácido borónico). El registro de cepas productoras de KPC es escaso en HULA ${ }^{25)}$ y la presencia de metaloenzimas en enterobacterias no había sido descrita hasta el momento.

$P$ aeruginosa es un conocido patógeno intrahospitalario, algunos estudios la reportan en un $10 \%$ aproximadamente en pacientes adultos con ITUAC y posee un alto riesgo de producir urosepsis ${ }^{(3)}$. Recientemente, Serrano et al. (26) describieron cepas de $P$. aeruginosa productora de metalo- $\beta$-lactamasa del tipo VIM-2 de origen urinario en pacientes adultos con sepsis en la emergencia de adultos del HULA. Del mismo modo, en este trabajo $P$. aeruginosa y A. baumannii mostraron una pobre susceptibilidad a cefalosporinas y carbapenemos y, fenotípicamente, se les detectó la producción de metalo $\beta$-lactamasas, además de presentar corresistencia a otros antibióticos. Estos hallazgos son preocupantes, debido a que antibióticos de reserva, como los carbapenemos, utilizados para el tratamiento de infecciones graves producidas por microorganismos resistentes dejan de ser alternativas eficaces, especialmente cuando se utilizan en forma empírica en las ITU asociadas a los cuidados de la salud.

Otros agentes productores de ITUAC aislados en este estudio, en menor frecuencia, fueron los cocos Gram positivos $(15,2 \%)$ representados, principalmente, por especies de Enterococcus, seguidos por especies de Staphylococcus. Al igual que lo descrito en la literatura, estos microorganismos se aislaron comúnmente en la población anciana con sondajes prolongados ${ }^{(1,2,5)}$. Ambos géneros bacterianos presentaron un patrón de resistencia dirigido principalmente a $\beta$-lactámicos, pero conservaron la susceptibilidad a los glucopéptidos.

Por otra parte, el análisis de las relaciones genéticas de las cepas de E. coli y K. pneumoniae reveló que en ambos géneros bacterianos se identificaron dos grupos clonales principales, los cuales se concentraron mayoritariamente en la unidad de trauma shock de la emergencia de adulto. De igual forma, el resto de bacterias Gram negativas multirresistentes fueron aisladas en pacientes recluidos en esta misma unidad. Esto indica que, desde el punto de vista de la epidemiología molecular, esta área se comportaría como el principal nicho para el intercambio y recombinación de genes codificantes para BLEE y carbapenemasas en bacterias Gram negativas, y representa el área de mayor riesgo y distribución de clonas multirresistentes. 
Es importante señalar que el corto periodo de estudio, aunado a la administración de antibióticos de amplio espectro a los pacientes antes de la recolección de la muestra clínica, pudieron ser factores limitantes que impidieron ampliar sustancialmente el perfil microbiológico de las ITUAC. Sin embargo, los resultados obtenidos representan un aporte valioso para establecer parámetros de referencia local, dirigidos a mejorar la calidad de la asistencia sanitaria en el HULA.

En conclusión, aun cuando las especies de Candida fueron los agentes más frecuentemente aislados en las ITUAC en el HULA y que estas presentaron una excelente susceptibilidad a los antifúngicos, otros agentes etiológicos como enterobacterias, bacilos Gram negativos no fermentadores y especies de Enterococcus y Staphylococcus destacaron por presentar perfiles de multirresistencia complejos, especialmente a los antibióticos $\beta$-lactámicos. Esta situación es alarmante, no solo porque las opciones terapéuticas para el tratamiento de las ITUAC son insuficientes, sino también porque las alternativas terapéuticas, como los nuevos glucopéptidos, colistina o tigeciclina, no son de acceso fácil por su elevado costo y escasa disponibilidad en el país. En consecuencia, urge implementar programas de prevención y control de la diseminación de clonas bacterianas multirresistentes, así como crear estrategias que permitan racionalizar el uso de los antimicrobianos.

Del mismo modo, se evidencia la necesidad de adoptar criterios que justifiquen la colocación de un catéter vesical y la duración del mismo. Además de implementar protocolos y prácticas rigurosas de higiene para la instalación, cuidado y mantenimiento del paciente cateterizado, con el fin de disminuir la incidencia de las ITUAC en los servicios de Medicina Interna del HULA.

Contribuciones de autoría: PQM y MA: diseñaron el estudio, analizaron clínica y epidemiológicamente los pacientes, realizaron la discusión crítica y la escritura final del manuscrito. AFC e IL procesaron las muestras y analizaron las cepas fenotípica y molecularmente. Todos los autores revisaron y aprobaron la versión final del manuscrito.

Fuentes de financiamiento: este trabajo fue financiado por el Consejo de Desarrollo Científico, Humanístico, Tecnológico y de las Artes de la Universidad de Los Andes, código: ADG FA-02-97-07 y FA-572-14-07-EE y Fundación Empresas Polar código: 140275.

Conflictos de interés: los autores declaran no tener ningún conflicto de interés.

\section{REFERENCIAS BIBLIOGRÁFICAS}

1. Flores-Mireles AL, Walker JN, Caparon M, Hultgren SJ. Urinary tract infections: epidemiology, mechanisms of infection and treatment options. Nat Rev Microbiol. 2015;13(5):269-84. doi: $10.1038 /$ nrmicro3432

2. Nicolle LE. Catheter associated urinary tract infections. Antimicrob Resist Infect Control. 2014;3:23. doi:10.1186/2047-2994-3-23

3. Pigrau C. Infecciones del tracto urinario nosocomiales. Enferm Infecc Microbiol Clin. 2013;31(9): 614-24. doi: 10.1016/j.eimc.2012.11.015

4. Horan TC, Andrus M, Dudeck MA. CDC/NHSN surveillance definition of healthcare-associated infection and criteria for specific types of infections in the acute care setting. Am J Infect Control. 2008;36(5):309-32. doi: 10.1016/j.ajic.2008.03.002

5. Jacobsen SM, Stickler DJ, Mobley HL, Shirtliff ME. Complicated catheter-associated urinary tract infections due to Escherichia coli and Proteus mirabilis. Clin Microbiol Rev. 2008;21(1):26-59. doi: 10.1128/ CMR.00019-07
6. Trautner BW. Management of catheter-associated urinary tract infection. Curr Opin Infect Dis. 2010;23(1):76-82. doi: 10.1097/ QCO.0b013e328334dda8

7. Clinical and Laboratory Standards Institute. Performance Standards for Antimicrobial Susceptibility Testing; Twenty-Fifth Informational Supplement. CLSI Document M100-S25. Clinical and Laboratory Standards Institute, Wayne, PA.USA, 2015.

8. Guevara A, de Waard J, Araque M. Detección del gen bla $\mathrm{VIIM}-2_{2}$ en cepas de Pseudomonas aeruginosa productoras de metalo b-lactamasa aisladas en una unidad de cuidados intensivos en Ciudad Bolívar, Venezuela. Rev Chilena Infect. 2009;26(4):336-41. doi: 10.4067/S0716-10182009000500005

9. Martín-Lozano D, Cisneros JM, Becerril B, Cuberos L, Prados T, Ortíz $\mathrm{C}$, et al. Comparison of a repetitive extragenic palindromic sequencebased PCR method and clinical and microbiological methods for determining strain sources in cases of nosocomial Acinetobacter baumannii bacteremia. J Clin Microbiol. 2002;40(12):4571-5. doi:10.1128/ JCM.40.12.4571-4575.2002

10. De Lira MA, Flores A, Fragoso L, Oliva BY, López E, Márquez ML, et al. Infecciones del tracto urinario asociado a catéter vesical. Áreas de cirugía y medicina interna de dos hospitales del sector público. Enf Inf Microbiol. 2012;33(1):13-8.

11. Duszynska W, Rosenthal VD, Szczesny A, Woznica E, Ulfik K, Ostrowska E, et al. Urinary tract infections in intensive care unit patients - a single-centre, 3-year observational study according to the INICC project. Anaesthesiol Intensive Ther. 2016;48(1):1-6. doi: 10.5603/AIT.2016.0001

12. Dougnon $\mathrm{T}$, Bankole $\mathrm{H}$, Johnson $\mathrm{R}$, Hounmanou G, Toure I, Houessou $\mathrm{C}$, et al. Catheter-associated urinary tract infections at a hospital in Zinvie, Benin (West Africa). Int J Infect. 2016;3(2):e34141. doi: 10.17795/iji34141 
13. Kanj SS, Zahreddine N, Rosenthal VD, Alamuddin L, Kanafani Z, Molaed B. Impact of a multidimensional infection control approach on catheter-associated urinary tract infection rates in an adult intensive care unit in Lebanon: International Nosocomial Infection Control Consortium (INICC) findings. Int J Infect Dis. 2013;17(9):e686-90. doi: $10.1016 /$ j.ijid.2013.01.020

14. Meddings J, Rogers MA, Krein SL, Fakih MG, Olmsted RN, Saint S. Reducing unnecessary urinary catheter use and other strategies to prevent catheter-associated urinary tract infection: an integrative review. BMJ Qual Saf.2014;23(4):277-89. doi: 10.1136/bmjqs-2012-001774

15. Tayal RA, Baveja SM, De AS. Analysis of biofilm formation and antibiotic susceptibility pattern of uropathogens in patients admitted in a tertiary care hospital in India. Int J Health Allied Sci. 2015;4(4):247-52. 10.4103/2278344X.167648

16. Al-Hazmi H. Role of duration of catheterization and length of hospital stay on the rate of catheter-related hospital-acquired urinary tract infections. Res Rep Urol. 2015;7:41-7. doi: 10.2147/RRU.S75419

17. Liedl B. [Catheter-associated urinary tract infections]. Urologe A. 2015;54(9):1301-8. Alemán. doi:
$10.1007 / \mathrm{s} 00120-015-3912-2$

18. Vyawahare CR, Gandham NR, Misra RN, Jadhav SV, Gupta NS, Angadi KM. Ocurrence of catheter-associated urinary tract infection in critical care units. Med J DY Patil Univ. 2015;8(5):585-9. doi: 10.4103/09752870.164974

19. Esquivel CG, Barbachano E, Avila HG, Celis F, Alonso SE, Martínez G. Perfil microbiológico en infección urinaria asociada a catéter vesicouretral. Medicrit. 2007;4(3):59-65.

20. Chincha O, Cornelio E, Valverde $\mathrm{V}$, Acevedo M. Infecciones intrahospitalarias asociadas a dispositivos invasivos en unidades de cuidados intensivos de un hospital nacional de Lima, Perú. Rev Peru Med Exp Salud Publica. 2013;30(4):616-20.

21. Deorukhkar SC, Saini S. Medical device-associated Candida infections in a rural tertiary care teaching hospital of India. Interdiscip Perspect Infect Dis. 2016;2016:1854673. doi:10.1155/20g16/1854673.

22. Flores-Carrero A, Paniz-Mondolfi A, Hernández E, Araque M. Candida pelliculosa blood infection in a neonate patient from Mérida, Venezuela. Intern J Adv Cases Reports. 2015;2(11):717-20.

23. Mishra M, Agrawal S, Raut S, Kurhade AM, Powar RM. Profile of yeasts isolated from urinary tracts of catheterized patients. J Clin Diagn Res. 2014;8(2):44-6. doi: 10.7860/ JCDR/2014/6614.4003

24. Girlich D, Poirel L, Nordmann P. Value of the modified Hodge test for detection of emerging carbapenemases in Enterobacteriaceae. J Clin Microbiol. 2012;50(2):477-9. doi: 10.1128/JCM.05247-11

25. Labrador I, Araque M. First description of KPC-2-producing Klebsiella oxytoca isolated from a pediatric patient with nosocomial pneumonia in Venezuela. Cases Reports Infect Dis. 2014;2014:434987. doi:10.1155/2014/434987.

26. Serrano-Uribe R, Flores-Carrero A, Labrador I, Araque M. Epidemiología y caracterización molecular de bacilos Gram negativos multirresistentes productores de sepsis intrahospitalaria en pacientes adultos. Avan Biomed. 2016;5(1):26-37.

Correspondencia: Maria Araque Laboratorio de Microbiología Molecular. Departamento de Microbiologia y Parasitologia. Facultad de Farmacia y Bioanálisis. Universidad de Los Andes, Mérida 5101, Venezuela

Telefax: (+58) 2742403180

Correoelectrónico:araquemc@ula.ve

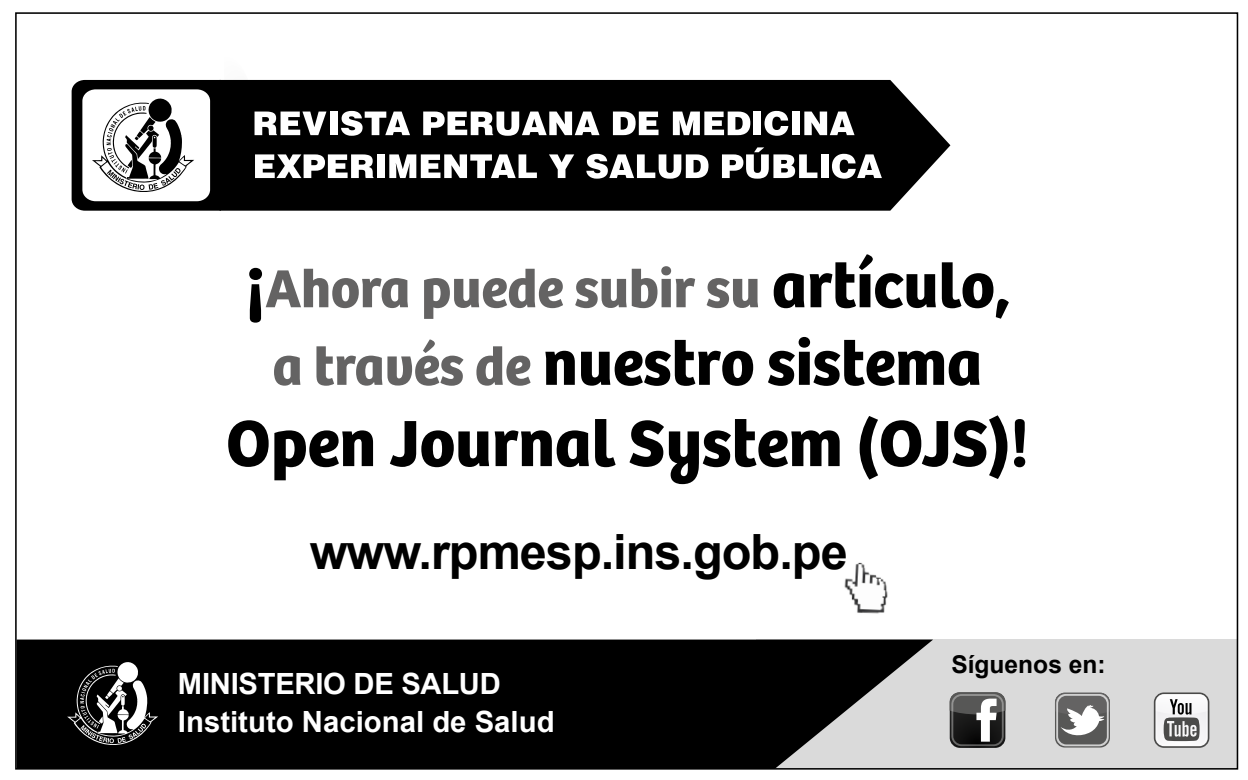

